\title{
Study on Crafts of High Voltage Cable Intermediate Joint
}

\author{
Dandan Su
}

Wuxi Institute of Arts \& Technology, Wuxi 214206, China

Sudandan001@163.com

Keywords: High Voltage Cable; Intermediate Joint; Craft

\begin{abstract}
High voltage cables intermediate joint craft is the essential technology during cable construction. In this paper, we firstly introduce the basic structure of high voltage cables, and then elaborate its production process and main precautions of high voltage cables intermediate joint, expecting that it is beneficial to the same industry.
\end{abstract}

\section{Introduction}

In the urban distribution network transformation and construction process, workers sometimes have to deal with high voltage cable intermediate joint. Generally speaking, compared with cable body, cable terminations and intermediate joints are weak parts, and most of the cable line faults happen at this section, so the quality of cable terminations and intermediate joints directly affects the safe operation of the cable lines. The slightest mistake will cause troubles, sometimes even cause widespread power outages and cause great loss to the country and the people's life and property. In this paper, we firstly introduce the basic structure of high voltage cables, and then elaborate its production process and main precautions of high voltage cables intermediate joint, expecting that it is beneficial to the same industry.

\section{Basic Structure of High Voltage Cables}

From inside to outside, high-voltage cables generally consist of nine layers of structure: polyethylene outer protective layer, armor layer, polyethylene inner protective layer, line package and filler layer, copper shielding layer, semi conductive layer, XLPE insulation layer, wire shielding layer and wire layer. The basic structure of high voltage cables is shown in Figure 1:

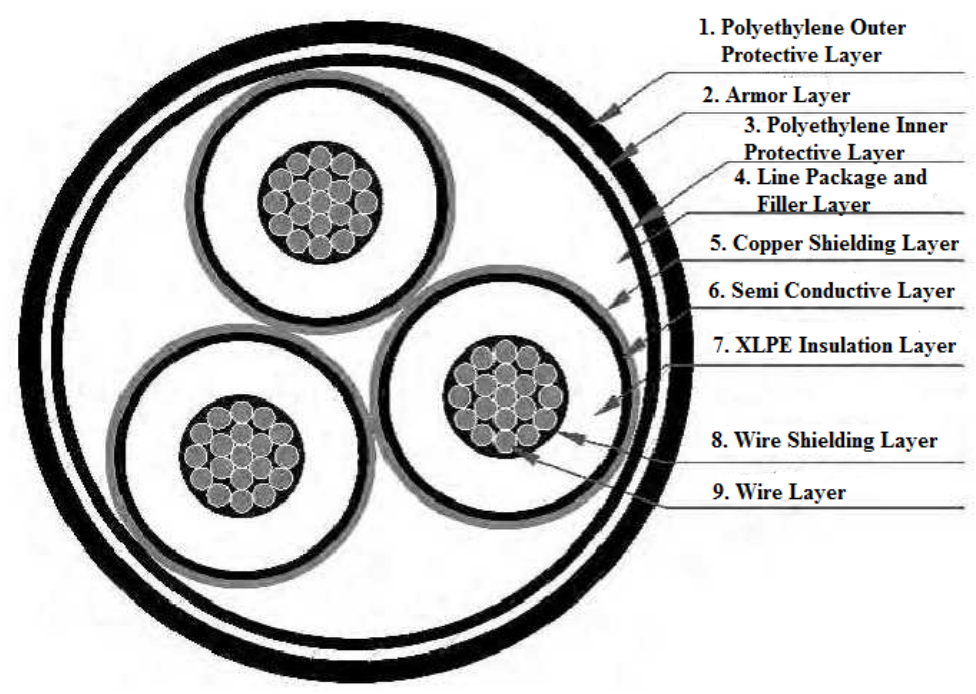

Fig. 1 Basic structure of high voltage cables

Polyethylene Outer Protective Layer. It can protect insulation layer from the harm of moisture and hazardous substances, so that maintain the stable performance of the internal insulation layer. 
Armor Layer. Armor layer can increase the mechanical strength of the cable; improve the anti-corrosion ability, compressive strength and other mechanical protection ability. In addition, armor also has a certain resistance to external force, and prevents animal bite, to prevent the power transmission problems of armor.

Polyethylene Inner Protective Layer. As similar as polyethylene outer protective layer, polyethylene inner protective layer can keep insulation layer away from water, air and other objects, avoiding moisture and mechanical injury on internal insulation layer, and meanwhile plays the role of protecting the cable core.

Line Package and Filler Layer. Line package and filler layer is used to protect the cable core, and keep fastening between cables. It organizes several cable cores into circular wire rod, for the convenience of packaging and cabling.

Copper Shielding Layer. The so-called shielding in terms of cable structure virtually means a measure to improve the electric field distribution, that is, to restrict the strong electric field produced when cable is working within shielding layer, so as to prevent the interference of strong electric field around on the transmission current in the cable. Therefore, if this copper shielding layer in cable doesn't exist, then insulation breakdown between core and core will be more likely to happen.

Semi Conductive Layer. There may be clearance in insulation surface or protective layer, which is one of the main factors causing partial discharge. So people cover a shielding layer of semi conductive materials, which has a good contact with the shielded layer, so that avoid the partial discharge between insulation layer and protective layer.

XLPE Insulation Layer. XLPE is short for cross-linked polyethylene, which is made of insulating materials. XLPE has characters of high breakdown strength, high insulation resistance, low dielectric loss, excellent tree discharge- resistance performance and long insulation performance period, etc.

Wire Shielding Layer. Wire shielding layer can improve the electric field distribution. The cable conductor is hinged with a plurality of wires, forming air gap between insulation layer, and its surface is not smooth, so it will cause the concentration of electric field. Cover a shielding layer of semiconductor materials on the surface of the conductor, which has good contact with insulation layer, so as to reduce the probability of occurrence of partial discharge. This layer is called inner shielding layer.

Wire Layer. The main function of wire is to transfer current. In order to reduce line losses and voltage drop, people generally use metal materials with high conductivity, and meanwhile consider the mechanical strength, price, source of materials.

\section{Production Process of High Voltage Cables Intermediate Joint}

There are many types of production process of high voltage cables intermediate joint. Here we take heat the relatively common shrinkable cable joints for example, to make brief introduction.

Construction Preparation. (1) Main materials: cable head accessories and other main materials are provided by manufacturers, attaching with certificate and manual. The type, size, voltage level of the materials should meet the design requirements.

(2) Auxiliary materials: auxiliary materials mainly include soldering, welding oil, calico, emery cloth, wire connecting pipe cleaners, gasoline, grease ointment, etc.

(3) Main equipment: main equipment mainly include blowtorch, crimping pliers, steel tape measure, hacksaw, electric chrome iron, electric knife, pliers, screwdrivers, large plates, etc.

Operating Conditions. (1) Cable laying is completed, and pass the insulation resistance test.

(2) The workplace environment temperature is above $0^{\circ} \mathrm{C}$, and the relative humidity is below $70 \%$. Operation in rainy, foggy, or windy days is prohibited.

(3) The construction site should be clean, spacious and well-lit. Construction site should be equipped with $220 \mathrm{~V}$ AC power.

Technological Process. The technological process of high voltage cables intermediate joint is shown in Figure 2: 


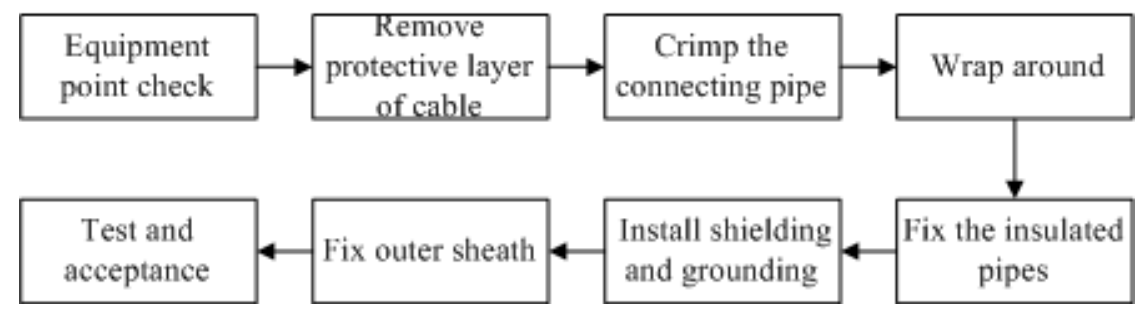

Fig. 2 Technological process of high voltage cables intermediate joint

(1) Equipment point check: unpack and check out whether the material objects are consistent with the information on packing list, in terms of quantity and quality, and examine if there is any abnormal phenomenon of their appearance.

(2) Remove the protective layer of cable: remove the copper shielding layer and semiconductor layer, as shown in Figure 3:

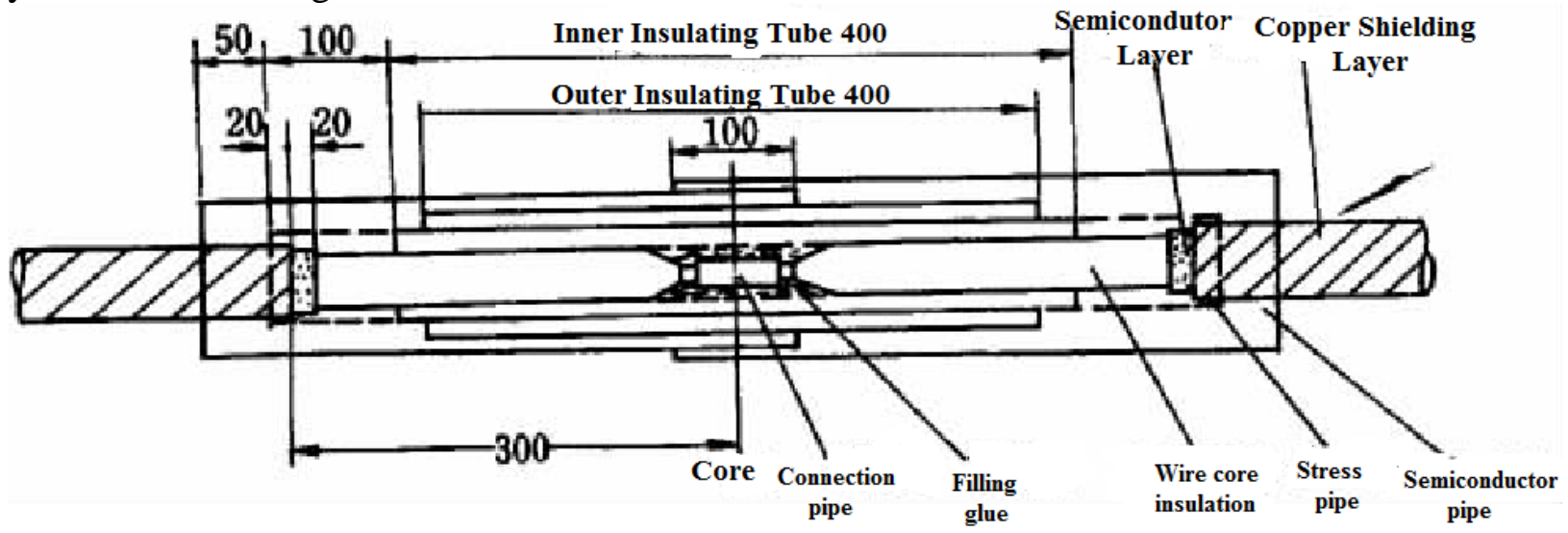

Fig. 3 Remove the protective layer of cable

(3) Crimp the connecting pipe: when manufacturing of high voltage cables intermediate joint, use conductor connection fittings to connect both ends of the cable wire core, so that the cable core is conducted.

(4) Wrap around the semi conduction band and filling glue: wrap around the semi conduction band on the surface of connecting pipe, and lap with both side of inner semiconducting shield layer, ensuring the continuity of inner semiconducting shield layer. Fill the semi conduction band layer with filling glue, with above $3 \mathrm{~mm}$, so that restore insulation characteristics of the primary insulation layer.

(5) Fix the insulated pipes: place the composite pipe between two stress pipes, and then heat it from the middle to both ends until it is fixed. After wrapping around waterproof glue at the composite tube ends, wrap around semi conduction band on the waterproof glue, with the two ends of the copper shielding layer and semi conductive layer overlapping composite pipe, for the recovery of semi conductive shielding layer.

(6) Install shielding and grounding: bundle up the copper screen with the copper shielding layer at both ends of connector and weld it; then curl up with ground wire and tie the core wire, with both ends welded on armor, so as to restore cable grounding.

(7) Fix outer sheath: after fixing the outer sheath, install shrink outer sheath tube or metal sheath tube. When the metal sheath tube and Sealing is well installed, the protective layer of the cable will be restored.

(8) Test and transmission operational acceptance: measure the insulation resistance, primary insulation resistance while transferring should be above $2500 \mathrm{M} \Omega$. Outer sheath and the inner sheath insulation resistance are above $0.5 \mathrm{M} \Omega / \mathrm{kM}$ (by $5000 \mathrm{kV}$ megger). After passing the test, check the cable, do security checks before transmission. During the operation process, strengthen the monitoring of current and voltage. 


\section{Precautions of High Voltage Cables Intermediate Joint Craft}

Creeping discharge at the electrode edges is more likely to happen than breakdown of the dielectric layer. So in the production process of cable intermediate joint, we should pay attention to some precautions as follow:

(1) Cable stripping: be careful that when strip one structural layer, do not harm other structural layers, so as not to cause problems for the future safe operation. The precautions while stripping are: should not hurt copper shielding layer when stripping inner liner; should not hurt outer semi conductive shielding layer when stripping copper shielding layer; copper shielding band cannot be loosed, and make sure that there is not rake angle at cut position; should not hurt main insulation layer when stripping outer semi conductive shielding layer; should not cable core when stripping main insulation layer. The outer semi conductive shielding layer should be 45 degrees down, and polished with fine sandpaper; main insulation layer should be cut until the inner semiconducting shield layer exposes an appropriate length, so that the inner semi conductive shielding layers at both ends of wire connecting tube are connected.

(2) Cable core connection: people generally adopt crimping method to achieve cable core connection. If crimp tube inner diameter and wire line core miss-match, the excessive voids will greatly increase the joint resistance value, so high temperature and heat during normal operation could easily lead to aging of the main insulation breakdown. Ragged connecting pipe and wire core surface edges will easily lead to tip discharge breakdown because of electric field concentration.

(3) Cleaning: craft of high voltage cable intermediate joint has strict cleaning requirements. In the production process, after the completion of each procedure, should use special cleaners to clean the cable joint surface, especially the trident point after ground welding, where should be carefully cleaned the remaining slag and the coke after using a blowtorch. In addition, should take care of some basic operations, e.g. do not wear dirty gloves; avoid the perspiration on hand or face catching on cable accessories, to make sure that every step of the production process are kept clean.

(4) Stress treatment: electrical stress control is important part in design of high-voltage cable accessories. Electrical stress control is to conduct control on cable accessories internal electric field distribution and electric field strength, that is, to implement appropriate measures so as to improve the operation reliability and service life of cable accessories.

(5) Steps treatment: after cable stripping, there will be one step between outer semi conductive shielding layer and main insulation surface. Should polish at outer semi conductive shielding layer cutting position with fine sandpaper, which is one of the most important measures of stress control.

(6) Sealing: sealing includes two meanings: one is moisture proof; another is to avoid the existence of air gap. At both ends of inner and outer protective sheath tube, should be sealed with sealant. When heat shrinkable tubing coated metal sealing parts such as connecting pipe, metal sheath, the metal parts should be heated to $60-70^{\circ} \mathrm{C}$, in order to obtain good sealing effect.

As described above, these precautions in field work are complementary, and each procedure is linked up and affected with others, so we should regard them as a continuous whole.

\section{References}

[1] Yian X. Opinions on Improving Quality of Fabricating Intermediate Joint of PVC High-voltage Power Cable. Nonferrous Metals Engineering \& Research, 2003.

[2] G.W. R, H.Q. S. Application and Popularization of Intermediate Joint Room of Cable Tunnel. Metallurgical Power, 2010.

[3] Z.G. W, L.J. Z. Construction of $500 \mathrm{kV}$ Cable Intermediate Joint. East China Electric Power, 2010.

[4] Ashibe Y, Takigawa H. Intermediate joint of superconducting cable: US, US20060162951 A1. 2005. 
[5] Wei P, Zhong-wen C, Shao-hong Q, et al. Reason Analysis of $10 \mathrm{kV}$ Cable Intermediate Joint Fault. Guangxi Electric Power, 2012. 